\title{
NEW METHOD FOR REACHING CONSUMERS OVER THE INTERNET: "SEARCH ENGINE MARKETING"
}

\author{
Çağrı Ergezer \\ Lecturer, University of Economy and Enterpreneurship - Faculty of Management , Jalal-Abad, \\ Kyrgyz Republic, cagriergezer@hotmail.com
}

\begin{abstract}
Internet has become a platform which reached millions of users momentarily with increased use, also become a place where people spent most of their time during the day by gaining consumer and potential customer ID in addition to just being ordinary Internet users. Search engines also have earned the distinction of being the preferred reference for users in the Internet sea which draws attention with usage rate and allowing you to easily reach the sought-after content where millions of content is added. Search engines has led to the birth of "Search Engine Marketing" concept which is gaining importance by considering the users as potential customers by companies who start to spend most of their time in different platform on the Internet or use search engines to find a subject we want to know, a music we like or any video or image. In the way for businesses to reach the consumers, search engines bring a different awareness in terms of competitiveness to marketing on the internet and are considered as an effective method that is preferred in today's e-marketing activities.
\end{abstract}

Although search engines for Internet users are frequently used as preferred web pages to access any information, at the same time it helps users to reach products or services over the internet with consumer identity. That is why as search engines for businesses and brands can be used in order to increase the possibility of awareness, listed as top rankings, or in a way partitioned according to the criteria specified on the web to attract the attention of consumers, the search engine can also become an effective marketing tool by using completely natural methods without paying any fees to ad units.

Especially in recent years search engine optimization which gained great importance and search engine marketing concepts have become concepts that should be examined in the framework of the marketing. That is why in this study the concept search engine optimization which is often preferred in e-marketing strategies was examined in detail in terms of businesses and brands, accordingly search engine marketing concept examined in theory in advertising and communication aspects and discussed in detail in terms of marketing factors.

Keywords: Search Engine Marketing, Search Engine Marketing, SEO, SEM, Search Engine Optimization, E-Marketing

\section{INTRODUCTION}

Internet is a revolutionary invention of the twentieth century, and it became a significant extent that could be 
the name of the twenty-first century. It is so that internet is one of the most popular services that people use with the utilization rate in current conditions, it also earned the distinction of being an immense network with millions of sites and billions of content it has. Today internet become the preferred platform on access to information and allow users to be in communication with each other by being used at home, at work, at school, at the library and even in our mobile devices.

Users can spend the majority of their time in communicating, reaching content related issues they were curious, watch video, listen to music, perform shares, can establish virtual environment through social networks, play games, shop, learn new information, may perform organizing efforts by expressing their problems thanks to the internet-based applications. In such cases, the internet-based communication technologies are developing day by day and grow to reach a wider audience. The demand and competition experienced on the internet platform which a combination of facilities can be found also led to the creation of many new and different strategies in terms of communication and noticeability. One of these Internet-based strategy which is increasing the noticeability for users by standing out from competitors is additionally specified as "search engines".

Search engines that makes it easy to reach the content by users which they are looking for in the internet environment in the most effortless and accurate way are preferred as the map on the web and they are the most common platforms that are being visited by users according to the researches. Because it is pretty hard, or even it is impossible for a user to reach the information he or she looks for from the billions of contents. The basic logic of the search engines which are created to close the gap at this point is to offer different alternatives to the visitors to present the wanted content in the most ideal, correct and quick way.

The mentioned visitors prefer search engines with their identity of being Internet users in order to find and reach the visual, audio, literary, moving information as well as they search and reach the product or service they are looking for with their consumer identity. Therefore, the opt for the search engines of the user groups who spend most of their time on internet platform to reach the product, service and brands they are looking for led the businesses, brands and marketing managers to develop a number of strategies which will enable them to be noticed. The "Search Engine Marketing" concept which is one of the search-based marketing strategies considered at this point and put into practice that will allow businesses to stand out from their competitors by facilitating the search and access to the product, service and brands for users who prefer the search engines.

In this study, the emergence of the concept of search engine, its evolution over the years and importance of search engines and how they become popular has been examined from the conceptual point of view, as well as proposals has been performed after detailed investigation about how businesses evaluate the search engines in terms of reaching to consumers, search engine marketing concept and preferred marketing strategies along with it.

\section{SEARCH ENGINE CONCEPT AND HISTORY OF SEARCH ENGINES}

Inherently human is equipped with curiosity and motivation for learning and has a tend to understanding, comprehension and access to information. In past people used to use printed materials such as books, magazines, encyclopedia but now have trend to opting search engines for their curiosity impulse and in the context of access to information with the expansion of the internet. Because the information is a lot more in the internet ocean, and in a more accessible form. That is why it can be faster and effortless to access the information sought through internet. In this context, internet users can reach to the information on the internet via search engines, and have the opportunity to compare information with different versions of content. Thus the user can be luckier according to conventional methods at the point of obtaining information on various diversity.

The concept of search engine can appear in different ways in terms of its definition. According to one of these definitions, the search engine is a mechanism used to search for content that exists on the internet. Search engine is defined as a query and the data acquisition mechanism that is comparing each of the queries of information and records reached as output, makes the necessary procedures for the admissibility of this query and consequently providing the high performance for data seized (Rowley, 2000:27).

According to another definition the search engine is the process of presenting the output to user by the comparison with the content found on the internet comparison with the content found on the internet and by analyzing the search in the most appropriate basis. These information matches with web sites, URL of the page indicated by keywords or phrases, in other words with codes and links that creates the page (Ledford, 2007: 5).

According to Gordon the search engine; a program for listing the web pages and storing the important 
information process in the database (Gordon, 2002: 218). According to this it is the case that reflecting the list of matching data to the user screen as a result of overlapping of search terms present in the system and data in the database.

As it can be seen, it is possible to come across to a few different definitions that are similar to each other. But before making a general definition by taking the advantage of all description it would be more appropriate to explain the concepts that make up the concept of search engine. These consists of three elements including: reference, organic reference and position. The reference is being mentioned as the mean to make reference to something with its dictionary meaning, and can be defined as finding the related sources with searched keywords. The organic reference is specified as the listing of the pages of the internet without paying any fees to the search engine operators. The position is defined as listing the pages found as a result of search results and their position in these lists (Gökaliler, 2010: 118).

By taking the above three components into account, it will be possible to define the search engine concept as listing process resulted after internet users use the keywords to query the information they are looking for on databases hosted on the internet and which are coupled with results from the reference by the query.

When we take a look to the history of the concept of search engine that has a history of nearly 25 years the first search engine system in a simple manner was implemented by Alan Emtage in 1990. Archie called search engine were listing the suitable results according to the searched word by scanning the contents of existing files by using FTP protocol instead of WWW protocol which was not being used yet in that time. Even the Archie system did not have highly qualified algorithms of today's search engines, it was a fundamental process for that period in terms of processing the search engine logic of today's. Immediately after Archie, using the search engines named as Gopher and Veronica has created environment to the emergence of needs of search engine in a professional sense. Because the situation of proliferation of the internet to home users instead of only formal institutions, the introduction of the WWW protocol and increase in Internet penetration made it necessary (Michael \& Salter, 2003: 2).

In the year of 1993 algorithms close to the working principle of today's search engine began to take shape and Wandex search engine began to webcast. In 1994 Yahoo began to webcast as a search engine which is capable of professional manner indexing and can be managed by people. In that periods, crawler, spider programs which are capable of collecting information by visiting the website has not been created yet and search engines based on the personal efforts were not sufficient to index the web content exponentially increasing. Nevertheless, Yahoo was the most preferred search engine of its kind with its ease of use and simple interface options. The years of 1994 - 1995 were the years in which also search engine trials was high. Many search engines based on different ideas, listing by using different algorithms became operational during this period. In 1995 Www Worn could reach 110 thousand websites, in 1997 WebCrawler managed indexes reaching over 2 million websites (Thomas, 1998: 144).

Alta Vista search engine opened in 1995 has managed to reach many more website than its competitors and has become quite popular with its spider and crawler software. HotBot search engine followed the Alta Vista at the same period and Microsoft that has a large share in the IT sector has developed the Bing search engine by taking part in the sector with the spread of Internet sites. MSN Search search engine that began to operate in 1998 used the same name since 2009 and continued to serve with its new name Bing after this date (Michael \& Salter, 2003:2).

Today most preferred search engine Google became operational in 1998 by Larry Page and Sergy Brin. About a year later, Google was among the top 100 websites. It continuously improved the concept of search engine, strengthen infrastructure with new investments and continued to protect its rank by seizing the leadership (Rognerud, 2008: 3). Today it is possible to say that many search engines operate in different categories.

\section{SEARCH ENGINE OPTIMIZATION}

Before explaining the concept of search engine marketing (SEO), it is necessary to examine the reasons for starting to use the search engines for marketing purposes. Because search engines are a new digital marketing tools and they gained their importance due to development of Web technology, become available to larger masses and opt for search engines in the process of access to information, requests of websites through search engines for access to a wider audience.

The concept of Search Engine Optimization (SEO) can be defined in several different ways. According to this the search engine optimization a set of studies to be listed in the top row in the list that reflected in the results according to searchable keywords (Davis, 2006: 2). According to this it can be said that the search engine optimization concept is the efforts to rank higher via search engines to attract the attention of visitors 
to increase the web traffic.

According to other similar definitions the search engine optimization can be defined as the process of the search engines to find your website (Kay 2007: 40 \& Malaga 2008,:149). Therefore, it can be said that it is the whole efforts to be listed in the search engine databases.

At the same time another definition, it is defined as all the activities which allow websites or contents to be listed in the top in queries held on search engines (Yalçın and Köse, 2010: 488).

Unlike other definitions search engine optimization is the art of achieving web site popularity which they cannot achieve with natural ways but by using natural-looking methods. This web popularity also brings the increase in the ranking in search engines too. In this way generated content can listed in the upper row compared to other competitors in search engines and thus will reach a wider audience with increased possibility of being noticed by visitors (http://seohocasi.com , 2010).

According to another definition the SEO is mentioned as applications that are attracting visitors by listing the sites or contents in free, editorial, organic search results, and is indicated as increasing efforts in free and natural way unlike paid search engine to be listed in the top row (http://searchengineland.com). Therefore, it should not be confused with paid search engine advertising.

Based on the definition of all three explanations it is possible to define the search engine optimization (SEO) as the whole process to ensure your content to be listed in the upper row in the eyes of the search engines in order to attract organic visitors to your website or your content. Also SEO include in site and off site arrangements which are very different from the paid search engine ads allowing you to be listed in the top row and include applications that allow your content to earn value with these arrangements.

\section{RELATIONSHIP OF SEARCH ENGINES AND CONSUMER BUYING BEHAVIOUR}

The spread of Internet use gave birth to an audience using the internet in a specific part of the day and attracted the attention of business with their consumer identity. The increasing number of Internet users has launched a new era in consumer communications in terms of businesses and brands. Reasons such as increasing awareness of consumers, insensitivity to their marketing efforts conducted through traditional media tools, decreases occurred in the use of traditional media tools restricted company and brands to reach the consumer. Due to these causes businesses and brands have begun to prefer internet that is becoming important day by day in order to reach the consumer in the marketing context.

At the same time internet become a platform which consumers use it to achieve the products and services, communicating with the brands and businesses, compare products and services as well as a platform where consumers search for information.

Consumer decision-making process has begun to show some differences with the developing Internet technology. Consumers before the Internet used to feel the need to physically walk around the shops in the investigation of the products and preferring the traditional media during the access to information process regarding the products or services. After internet this has tended to change and consumers have started to prefer internet in the process of access to information. Therefore, the cost and hassle of access to information largely be eliminated and internet has been playing an active role in consumer purchase decision process (Mahajan and Mind, 2001: 173).

According to Solomon, the consumers tend to conclude need first, access to information relating to the product or service, evaluation of different options, deciding specified product or service and purchasing behavior in the purchase decision-making stage for a product or service (Solomon, 2007: 305). In this regard, the internet, provides simpler solutions when compared with physical efforts by allowing more effortlessly traversing to consumers in all these stages. Also studies conducted proves these situations. Namely; according to a study conducted in the USA in 2008 83\% of a large portion of Internet users are affected by the products and services on the internet. In the same study $70 \%$ of consumers perform collection activities about the related product or service on the internet before buying the product or service (Werbler and Harris, 2008).

Although internet is providing advantages such as time saving, reach to more information in less time, more choices and reach to more information, the consumers often reluctant about shopping on the Internet and prefer traditional face to face shopping methods (Lin, 2007: 439). Chief Marketer research company conducted a research supporting this hypothesis in 2008; and concluded that 80 million consumers in the United States doing research about the service, product and brand via the Internet but perform the purchase process through stores (Balzer, 2008). 
At the same time ease of consumption which is increasingly important in recent years for consumers has become a phenomenon and it has effect on the purchasing behavior of consumers and becoming the most basic expectations for consumers who are in online buyer position. According to these two different elements that attract the attention of consumers is concerned on the internet. The first one is to meet the consumer's expectations and the second one is the positive value it provides for them. Ease of consumer policy is the expectation to meet with the most affordable price and without much hassle on the internet for online consumers. Internet consumers leave the websites quickly and directed to a different website when they have difficulty in finding the products they want to purchase, see prices that exceeds the expectations, payment options that are unsafe or inadequate, their desired delivery options are different or too long. For instance, consumers who want to buy any book may not want to own that product by touring bookstore to bookstore to compare the prices however internet consumers may decide to purchase a product they want to buy by making price comparison in a few minutes by examining the websites. In summary it will be possible to say that the ease of consumption is one of the most important points which enticed ordinary consumers in electronic commerce. The positive value of the internet for consumers is; linked with product or service preferences of consumers with their identity on the internet to be determined. For instance, personalized internet ads can appear as product or service-related about the things we are looking on internet (Ergezer, 2014).

According to a similar study conducted by ComScore a large proportion of $63 \%$ of Internet users prefer search engines about products or services in the information retrieval process and perform their purchase from the shopping sites that directed by search engines (Balzer, 2008).

When we examine in terms of the business it is known that search engines are correcting errors, directing visitors and provide the most accurate results at the search stage. Today, many popular search engines make error corrective recommendations during search and perform the format and type propositions by listing the search results in types of audio, video and photo not only the search results in a text type. Even today's search engines can be personalized with user information and achieves optimal search results by matching the user's demographics with their sought-after information. In this way search engine recognizes the user and is able to analyze individual properties with searches over the Internet (İçil, 2008: 26). In this case also it is possible to influence the consumer purchase behavior. Because overlapping search results with personal information will direct consumers with product or service recommendations.

As a result, internet and search engines are able to influence purchasing behavior of consumers directly and indirectly, can direct them in the point of purchase. Therefore, businesses and brands can prefer search engines that are used extensively as a marketing tool and perform search engine marketing supporting study processes.

\section{SEARCH ENGINE MARKETING (SEM) CONCEPT}

The efforts to increase the search engine ranking in order to attract visitors to the web site content began to assume an important role in the Internet market in terms of marketing in time. Because in terms of businesses and brands internet media which hosts millions of visitors are not only a place where people spend their free time and only receive information, at the same time it is also become an area where people are looking for products or services, scan the information on the brand and model, try to access content on the company, compare their products or services with their consumer identity. Therefore, the request of businesses and brands to reach their potential customers in a simpler and more efficient way began to gain importance with search engine optimizations in terms of bringing the products and services to a wider consumer audience.

The concept of search engine marketing (SEM) put forward by Danny Sullivan for the first time in 2001 and define search engine optimization as directing the web content too indexes by combining paid listing methods and online marketing methods (Deitel, 2008: 11).

As definition the Search Engine Marketing concept is a set of comprehensive studies such as performance analysis, search engine optimization, Internet advertising to reach consumers directly which is allowing web pages that host products or contents to reach the target audience via search engines for people who prefer search engines. (Scott, 2009: 254)

According to another definition the search engine marketing is the process to make the internet content more accessible in search engines and referral sites, attracting the attention of visitors. In this process it is the process of the preparation of the content, improving visibility with search engine optimization, increasing the traffic to content with pricing per click (Michael \& Salter,2003:2).

The most important point to be considered in all definitions, search engine optimization (SEO) process is the 
basis of search engine marketing efforts. Because in search engine marketing process, it is aimed to increasing the visibility of web contents belonging to product, service, brand and business in the eyes of internet visitors, attracting more visitors to content and reach a wider audience. Therefore, search engines which gained prominence in the early 2000s has enabled more easily communication with consumers in terms of marketing.

On the basis of search engine marketing while there are fees paid for search engine optimization, paid impressions, paid link distributions, paid social media advertising, at the same time it is possible to say that there is free of charge works too. Businesses that create successful contents share their contents for free, attract visitors' attention without paying by using content marketing methods through social media channels.

The most important reason to use the search engine is the request to access to information. In this context as the sought information can be about a product or service, the internet users which are seeking information relating to the matters that businesses serve are at the same time the potential customers and company's target consumers too. With this reason businesses and brands may prefer the search engine marketing strategies in order to attract the consumer's attention in the searches related with their products or services. In addition, one of the most important factors in the choice of search engine marketing is the ability to reach consumers geographically with location based access and to make a partitioned internet marketing. In this way the searcher's location can be determined, and provided access to local businesses closest to them with matching search results with search words. Thus search engine marketing creates opportunities not only for large enterprises, at the same time it is preferred by small and medium-sized local businesses too (Gökaliler, 2010: 153).

At the same time in addition to geographic targeting it is possible to compartmentalize the consumers with many options such as gender targeting, targeting age groups, educational status during paid search engine marketing efforts processes. For instance, with the AdWords advertising system managed by Google many filtering options may be preferred such as keyword, geographic targeting, age, gender. In this way concerned consumers can be easily reached fictionalized marketing strategy. It is possible to see the similar application almost all the search engines in operation today. Filtering ability during the search results by compartmentalizing the user group is one of the most important elements of search engine marketing.

Almost every element in the search engine marketing efforts draws the attention of business with their ability to be measured (http://www.medianozz.com/sem/). Inability to measure lacking in traditional media has a negative impact on the marketing process. However, since search engine marketing is consisting of completely measurable elements; it is possible to make statistical measurements such as how many people were reached, how many people were included in the return, how many people have shown a buying behavior.

Today although paid search engine ads are basics of search engine marketing activity, the search engine marketing concepts and search engine ads may be diverging. Because, as you can see in Figure 1, businesses which prefer search engine ads takes the first three position in search engine ranking. When search results and directed web sites are examined, we can reach the conclusion that search engine ads belong to businesses and e-commerce web sites. That is why because search engine ads are preferred for the purpose of direct sales and marketing, it can enter into the limits of direct marketing. However, search engine marketing includes applications not only for direct sales at the same time too. In this context the content marketing emerges as one of the most preferred strategy in search engine marketing practices (Ergezer, 2014). Search Engine Marketing Professional Organization president Collin appropriately summarize this situation, at the same time define the search engine marketing concept as a mixture of search engine advertising and public relations. Through this definition titles that affect the consumers awakened curiosity in the eyes of consumers with the right message, the content which can be differentiated in the upper row; and also makes it easy to obtains the results desired with search engine marketing with the help of promotional mix elements such as advertising and public relations (Dysart, 2004: 27). 
Figure 1: Example of search engine advertising, http://www.google.com

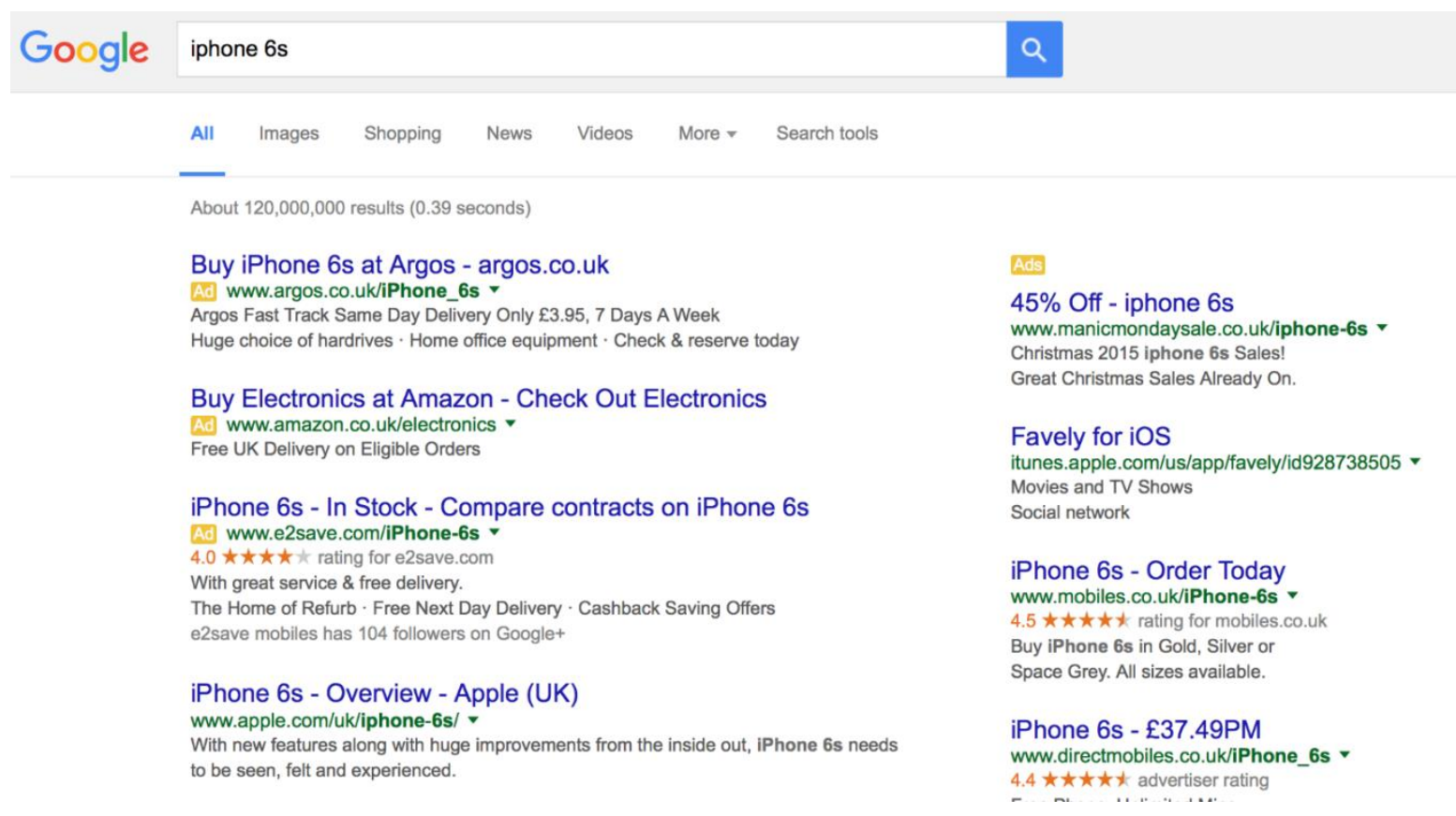

When we approach to the concept of search engine marketing in terms of consumers it is possible to see that consumers are hesitant in this marketing effort as in all marketing efforts. According to this the internet users can realize the listed paid and unpaid search results as a result of their search results. Search results that are listed in the top row with paid fees may remain insufficient compared to the listed unpaid organic search results from a consumer standpoint. Hence it will be wrong to adopt the search engine marketing activities as you can be listed in the top row by only paying the fee for search engines. A study that proves the case conducted in 2014 by Regalix Research. According to the survey organic search results are clicked on more by internet users when compared with the paid ads that are listed in the top row and they are preferred a lot more when compared with the paid ones in the process of reaching knowledge of products and services and purchasing process (Victor and Khan, 2014: 7).

When we examine the search engine marketing concept in terms of strategy; we can mention the existence of three methods as search engine marketing strategies, these are:

a) Search engine optimization

b) Search engine advertising (charged per click advertising / Pay-Per-Click /PPC)

c) Paid and free content marketing methods that support the search engine optimization

Search engine optimization is intended to provide to be more noticeable by search engine users and consist of paid and free effort aiming to appear in the top row in listed keyword-based search results. According to this search engine optimization process covers to make arrangements within the site, create quality content, get content links with comments made in quality blogs, perform link shares in forums and discussion groups, getting links named backlinks which allow directing to your web site or contents, share content links by getting profile links in web 2.0 web sites and adding your contents to bookmarking sites that are proposed by the search engines (Wueben, 2011: 130).

Search engine advertising can be defined as purchases aimed to be listed in the top row in search engines without the need for natural arrangements for attracting users in search engines to visit your website with per-click fee. This method is one of the cornerstones of search engine marketing however can be inadequate in influencing the consumer.

The content marketing methods can be defined as a process covering all activities related to not intended for direct advertising, raise awareness for company by engaging the audiences and beyond them, reach the target audience, establish a relationship with them, and creation of content to achieve measurable results by mobilizing them, detecting channels to publish the content, distribution and measure of content (Penpece 2013: 48). This process is one of the most important elements that support the search engine optimization. Because in search engines the attention of the target audience can be obtained with the preparation of quality remarkable, not intended for direct advertising content not only with listing in the top row. 
From all of these research and opinions, search engine marketing process can be defined as preparing quality ingredients intended to attract the attention of consumers with paid and free methods, the selection of the Internet channel supported with paid and organic work that provides to be listed in the top ranking in search engines and distribution and measurement of content.

\section{CONCLUSION}

As a result of the emergence of the Internet, the emergence of search engine concept, the increasingly widespread use of internet, increase of web content; search engines preferred in the process of access to information has gained an increasing importance. Internet has become a conduit where millions of users exist and has become a frequently preferred platform for businesses and brands in order to reach to consumers due to it is more functional when compared with traditional media channels. In this context businesses prefer to take the advantage of the potential offered by the internet and search engine marketing as possible, in this way is attempting to sell their product or service to potential customers by expanding the target consumers.

Within the framework of marketing one of the most ideal use of search engines is the concept of search engine marketing which began to gain importance by search engines are preferred often by visitors. In the context of reaching the consumer it can make more efficient partitioning with less cost when compared with traditional media channels with personal property positioning geographical positioning, demographic positioning options and therefore, it makes it easier to be contacted with the consumer audience in search results for products or services.

According to 2014 statistics $\$ 26$ billion has been spent to the search engine marketing strategy in the world and it is expected to reach $\$ 40$ billion as of the end of 2019. In this context, as search engine marketing can be preferred by big businesses and brands, at the same time it is also preferred by small and medium-sized enterprises. With these feature search engine marketing investments perhaps will have less investment than traditional marketing efforts however it will appear as a marketing activities that is preferred by small and medium-sized enterprises. While many of the predictions pointing that marketing efforts via traditional media will be more efficiently performed through digital platform in the near future, it is determined that search engine marketing will be the one which will be mostly preferred within these platforms.

Therefore, in the context of access to consumers via the Internet search engine marketing, identification of the target audience, segmentation of the Internet market has become one of the most effective marketing strategy of digital marketing in the context of increasing web site traffic. Groundbreaking search engine marketing increases success graph rapidly as it is measurable in terms of consumers, businesses and marketing dimension.

\section{REFERENCE LIST}

Harold Davis (2006), Search Engine Optimization, O'Reilly

Dysart J., (2004), "Search Engine Strategies", Information Today, Issue: September

Scott D. M. (2009), Pazarlamanın ve İletişimin Yeni Kuralları, Çeviri: Nadir Özata, İstanbul, Kapital Medya Hizm. A.Ş.

Victor B., Khan T., (2014) "State of Search Marketing 2014 Survey Report", Regalix Research, USA http://www.regalix.com/wp-content/uploads/2014/05/State-of-Search-Marketing-2014.pdf Access: 13.09.2015

Harvey M. Deitel (2008), AJAX, Rich Internet Applications, and Web Development for Programmers, Prentice Hall

Solomon R. M., (2007), Consumer Behavior, Buying, Having, and Being, Prentice Hall, 7th Edition, New Jersey

Werbler C., Harris C., (2008) Online Consumer Reviews Significantly Impact Consumer Purchasing Decisions, New Opinion Research Corporation Survey Finds http://www.reuters.com/article/idUS117426+24-Jun-2008+BW20080624 Access: 14.10.2015

Russel Kay (2007), Search Engine Optimization, Strategies\&Tactics, ComputerWorld, June 4

Gordon M., (2002), Robot Builder's Sourcebook: Over 2500 Sources For Robot Parts, McGraw- Hill Professional

Thomas B. J., (1998), The World Wide Web For Scientists\&Engineers: A Complete Reference For 
Navigating, Researching\&Publishing Online, IET

Balzer C., (2008) Searching for the retail value of SEM, http://multichannelmerchant.com/webchannelseo/searching-for-the-retail-value-of-sem-01122006/ Access: 10.11.2015

Wind Y., Mahajan V., (2001) Digital marketing: global strategies from the world's leading experts, John Wiley and Sons, ISBN 0471361224, 9780471361220

Lin, H.F., (2007), Predicting Consumer Intentions to Shop Online: An Empirical Test of Competing Theories, Electronic Commerce Research and Applications 6 (2007) 433-442, doi:10.1016/j.eleralp.2007.02.002. http://web.nchu.edu.tw/pweb/users/arborfish/lesson/8963.pdf Access: 11.10 .2015

Taban V. (2008), "Arama motorları ve gelecek", IP Magazine

Gökaliler, E., (2010), İnternet Reklamcılığında Yeni Bir Mecra: Arama Motoru Reklamcılığı, Basıımamış Doktora Tezi, Ege Üniversitesi Sosyal Bilimler Enstitüsü, İzmir

Rognerud, J., (2008), Ultimate Guide to Search Engine Optimization: Drive Traffic, Boost Conversion Rates and Make Lots of Money, Entrepreneur Press

Michael A., Salter B., (2003) Marketing Through Search Optimization: How To Be Found On The Web, Butterworth-Heinemann

Ergezer Ç., (2014) "İnternet Ziyaretçisinden, Online Müşteriye”, http://ergezer.net/internet-ziyaretcisindenonline-musteriye.html Access: 21.09.2015

Ergezer Ç., (2014) "Pazarlama Açısından SEO", http://ergezer.net/pazarlama-acisindan-seo.html Access: 21.09.2015

Penpece D., (2013), Dijital İçerik Pazarlaması, Karahan Kitapevi, Adana

Ross A. Malaga (2008), Worst Practices in Search Engine Optimization, Communications of the ACM, December, Vol.51, No.12

Steve Cooper, (2004) Search Engine Optimization, Entrepreneur, December, Vol. 32 Issue 12,

Rowley J., (2000). Product search in e-shopping: a review and research propositions, Journal of Consumer Marketing , 17 (2), 20-35.

Ledford J. L., (2007), SEO - Search Engine Optimization Bible: Search Engine Optimization Bible. John Wiley and Sons, ISBN: 978-0-470-17500-2

Wuebben J. (2011), Content is Currency: Developing Powerful Content For Web and Mobile, Nicholas Brealey Publishing, London - England

Yalçın N., Köse U., (2010), "Whats is Search Engine Optimization: SEO”, Procedia Social and Behavioral Sciences, Volume 9, Pages 487-493 http://ac.els-cdn.com/S1877042810022901/1-s2.0S1877042810022901-main.pdf?_tid=4cb57e70-e84f-11e4-9f0000000aacb362\&acdnat=1429638981_77b99a7311903fc0b6a6c0cb9b35cc02 Access: 19.08 .2015 (2010), "SEO Nedir, Nasıl Yapılır?" http://www.seohocasi.com/seo-nedir/, Access: 11.09.2015 (2010), "What is SEO / Search Engine Optimizatinon", http://searchengineland.com/guide/whatis-seo, Access: 10.10.2015

\subsection{1 .2025}

